\title{
SIGNED SUMS OF RECIPROCALS II
}

\section{Dedicated to George Szekeres on his 65th birthday}

\author{
R. T. WORLEY
}

(Received 5 December 1974)

\begin{abstract}
The author investigates $M(n, \alpha)=\min \left|\alpha-\Sigma \eta_{k} k^{-1}\right|$ where the minimum is over all sets of signs $\eta_{i}= \pm 1$ and shows $M(n, \alpha)<n^{-\frac{1}{2}(1-\varepsilon) \log _{2} n}$ for $|\alpha|<\frac{1}{2}(1-\varepsilon) \log n$.
\end{abstract}

In the previous paper it was shown that for given $\varepsilon>0$ there exists $n_{\varepsilon}$ such that for $n>n_{\varepsilon}$ it is possible to choose suitable signs $\eta_{k}, 1 \leqq k \leqq n$, such that

$$
\left|\sum_{k=1}^{n} \eta_{k} k^{-1}\right|<n^{-\frac{1}{2}(1-\varepsilon) \log _{2} n}
$$

where $\log _{2}$ denotes the base 2 logarithm. The aim of the present paper is to extend this result to make $\Sigma \eta_{k} k^{-1}$ close to numbers $\alpha$ not necessarily zero. The exact result obtained is:

THEOREM. For given $\varepsilon>0$ there exists $N_{\varepsilon}$ such that for $n>N_{\varepsilon}$ and real $\alpha$ with $|\alpha|<\frac{1}{2}(1-\varepsilon) \log n$ it is possible to choose signs $\eta_{k}, 1 \leqq k \leqq n$, such that

$$
\left|\sum_{k=1}^{n} \eta_{k} k^{-1}-\alpha\right|<n^{-\frac{1}{2}(1-\varepsilon) \log _{2} n}
$$

The method of proof is to split up the sum so that the following elementary result can be applied.

LeмmA 1 . Let $t_{1} \geqq t_{2} \geqq \cdots \geqq t_{\rho}$ be a sequence of positive real numbers with $t_{j} \geqq \frac{1}{2} t_{j-1}$ for $2 \leqq j \leqq \rho$ and let $\alpha$ be real. Then there exist signs $\varepsilon_{j}, 1 \leqq j \leqq \rho$, such that for $1 \leqq k \leqq \rho$

$$
\left|\alpha-\sum_{j=1}^{k} \varepsilon_{j} t_{j}\right|<\max \left(t_{k},|\alpha|-\sum_{j=1}^{k} t_{j}\right) .
$$

Proof. Set $\varepsilon_{1}$ to be the same sign as $\alpha$, so that (2) is satisfied with $k=1$. For $k \geqq 2$ we define $\varepsilon_{k}$ inductively to be the same sign as $\alpha-\sum_{j=1}^{k-1} \varepsilon_{j} t_{j}$. Since 
$t_{k-1} \geqq t_{k} \geqq \frac{1}{2} t_{k-1}$ it is clear that (2) holds when $t_{k-1} \geqq|\alpha|-\sum_{j=1}^{k-1} t_{j}$. On the other hand if $t_{k-1}<|\alpha|-\sum_{j=1}^{k-1} t_{j}$ then $\varepsilon_{1}, \varepsilon_{2}, \cdots \varepsilon_{k}$ are all of the same sign as $\alpha$ and

$$
\left|\alpha-\sum_{j=1}^{k} \varepsilon_{j} t_{j}\right|=|\alpha|-\sum_{j=1}^{k} t_{j} .
$$

COROLLARY. Let $t_{1} \geqq t_{2} \geqq \cdots \geqq t_{\rho}$ and $s_{1} \geqq s_{2} \geqq \cdots \geqq s_{\sigma}$ be sequences of positive numbers with $t_{\rho} \leqq 2, s_{1} \geqq 1, t_{j} \geqq \frac{1}{2} t_{j-1}$ for $2 \leqq j \leqq \rho$ and $s_{j} \geqq \frac{1}{2} s_{j-1}$ for $2 \leqq j \leqq \sigma$. Then if $\alpha$ is a real number such that $|\alpha|+2 \leqq \sum_{j=1}^{p} t_{j}$ there exist signs $\varepsilon_{j}, 1 \leqq j \leqq \rho$ and $\delta_{j}, 1 \leqq j \leqq \sigma$, such that

$$
\left|\alpha-\sum_{j=1}^{\rho} \varepsilon_{j} l_{j}+\sum_{j=1}^{\sigma} \delta_{j} s_{j}\right| \leqq s_{\sigma} .
$$

Proof. By the lemma we choose $\varepsilon_{j}$ to ensure $\left|\alpha-\sum_{i=1}^{p} \varepsilon_{j} t_{j}\right| \leqq 2$. Since $\sum_{j=1}^{\sigma} s_{j} \geqq 2$ a further application of the lemma gives the desired result.

The proof of the theorem hinges on constructing suitable sequences $s_{1}, s_{2}, \cdots, s_{\sigma}$ and $t_{1}, t_{2}, \cdots, t_{\rho}$ to make the left side of (3) of the same form as the left side of (1). To construct these sequences we need a few preliminary results.

Lemma 2. For integral $k \geqq 0, a>0$ there exist signs $\mu_{j}= \pm 1,0 \leqq j \leqq 2^{k-1}$ such that $s(a, k)={ }_{\text {let }} \sum_{j-0}^{2^{k}-1} \mu_{j}(a+j)^{-1}=2^{\frac{1}{2^{k(k-1)}} k ! \beta^{-k-1}}$ with $\beta \in\left[a, a+2^{k}\right)$.

Proof. For $k=0$ this is trivial, and for $k>0$ this follows from lemmas 1 and 2 of Worley (1976).

Lemma 3. For $b \leqq 16$ and $m>2^{b+2}$ let $f(x)$ be defined by $f(x)=$ $\left(2^{x-2} m^{x-1}\right)^{1 / x}-2^{x}$ for $2 \leqq x \leqq B=b-2 \log _{2} b$. Then $f$ is increasing.

Proof. Differentiating gives $f^{\prime}(x)=2 m 2^{-2 / x} m^{-1 / x}(\log 4 m) x^{-2}-2^{x} \log 2>$ $\left(2^{1-2 x^{-1}+(b+2)\left(1-x^{-1}\right)-\log _{2} b}-2^{x}\right) \log 2$ since $m>2^{b+2}$ and $x<b$. Thus $f^{\prime}(x)>0$ provided $h_{b}(x)>0$, where $h_{b}(x)=3+b-x-\log _{2} b-(b+4) x^{-1}$. Now $d h_{b}(x) / d x$ is positive for $2 \leqq x \leqq(b+4)^{\frac{1}{2}}$ and negative for $(b+4)^{\frac{1}{2}} \leqq x \leqq B$, sō it is only necessary to show $h_{b}(2)>0$ and $h_{b}(B)>0$. Plainly $h_{b}(2)=\frac{1}{2}\left(b-2 \log _{2} b-2\right)$ is positive for $b \geqq 16$ so it remains to consider $h_{b}(B)=3+\log _{2} b-(b+4) /\left(b-2 \log _{2} b\right)$. It is easily verified that $(b+4) /\left(b-2 \log _{2} b\right)$ is decreasing for $b \geqq 16$ and its value at $\boldsymbol{b}=16$ is $2 \frac{1}{2}$. Hence $h_{b}(B)$ is positive as required.

COROllary. Let $a_{1}=1, a_{2}=m^{\frac{1}{2}}-4$, and let $a_{i}$ be defined inductively for $3 \leqq j \leqq J=B$ as the greatest integer less than $\left(2^{i-2} m^{j-1}\right)^{1 / j}-2^{\prime}$ that is congruent to $a_{j-1} \bmod 2^{j-1}$. Then (i) $a_{j}<m$ and (ii) $a_{j-1} \leqq a_{j}$ for $2 \leqq j \leqq J$.

Proof. The inequality $2^{J-2}<2^{b}<m$ yields $2^{J-2} m^{J-1}<m^{J}$ which implies (i). The result (ii) is trivial for $j=2$ and follows from the lemma for $j \geqq 3$.

Lemma 4. Let $m, b, J, a_{1}, \cdots, a_{J}$ be as above and let $a \geqq a_{j}$. Then 


$$
\left(a+2^{j+1}\right)^{k}<2 a^{k}
$$

for integers $j \geqq 2, k \geqq 1$ satisfying $k<j$.

Proof. Since $k \leqq j$ it is only necessary to show $k \log \left(1+2^{j+1} a^{-1}\right)<\log 2$. Since $\log (1+x)<x$ and $a \geqq a_{j}>\left(2^{j-2} m^{j-1}\right)^{1 / j}-2^{j+1}$ it suffices to show

$$
1+j / \log 2<2^{b-i+2-(b+4) j^{-1}} .
$$

For $2 \leqq j \leqq(b+4)^{\frac{1}{2}}$ the rigth side of (5) is at least $2^{\frac{1}{2(b-4)}}$ and inequality (5) follows easily. For $(b+4)^{\frac{1}{2}} \leqq j \leqq J$ the right side of $(5)$ is at least $2^{2 \log _{2} b+2-(b+4) /\left(b-2 \log _{2} b\right)}<$ $2^{2 \log _{2} b}=b^{2}$ and again (5) follows.

LEMmA 5. If $m, b, J$ are as above, $a>m, 2 \leqq j \leqq J$ and

$$
a^{\prime}<\left(2^{j-2} m^{j-1}\right)^{1 / j}-2^{j-1} \text { then } s\left(a^{\prime}, j-1\right) \geqq \frac{1}{2} s(a, j-2) \text {. }
$$

Proof. Since $(j-1) ! \geqq(j-2)$ ! we have

$$
2^{\frac{1}{2}(j-1)(j-2)}(j-1) !\left(2^{j-2} m^{j-1}\right)^{-1} \geqq 2^{\frac{1}{2}(j-2)(j-3)}(j-2) ! m^{-j+1}
$$

which, by Lemma 2 , yields $s\left(a^{\prime}, j-1\right) \geqq s(a, j-2)$.

LEMMA 6. With the above notation

$$
s(a, J-1)<n^{-\frac{1}{2} \log _{2} n+7+3 \log _{2} \log _{2} n}
$$

where $a>n-2^{J+1}$ and $2^{b+3} \leqq n<2^{b+4}$.

Proof. By Lemma 2, since $a>\frac{1}{4} n$, we have $s(a, J-1)<$ $2^{\frac{1}{2}(J-1)(J-2)}(J-1) ! 4^{\prime} n^{-J}$. As $J<\log _{2} n$ it is easily seen that

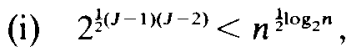

(ii) $(J-1) !<\left(\log _{2} n\right)^{\log _{2} n}=n^{\log _{2} n \log _{2} n}$,

(iii) $4^{J}<n^{2}$,

(iv) $n^{J}>n^{b-2 \log _{2} b-1}$, and

(v) $b-2 \log _{2} b-1>\log _{2} n-5-2 \log _{2}\left(\log _{2} n-4\right)$.

Combining these inequalities yields (6).

We are now in a position to prove the theorem. We assume $n \geqq 2^{19}$ without loss of generality, define $b \geqq 16$ by $2^{b+3} \leqq n<2^{b+4}$, set $m=n-2^{b+1}$ and define $B, j, a_{1}, \cdots, a_{J}$ as above. We also set $a_{J+1}$ to be the greatest integer less than or equal to $n$ that is congruent to $a_{J} \bmod 2^{J}$. Since $a_{J}<m$ and $J \leqq b<b$ we clearly have $a_{J+1}>a_{J}$. Define $r_{j}$, for $2 \leqq j \leqq J$ by $a_{j+1}=a_{j}+r_{j} 2^{j}$.

Consider.firstly the collection of sums

$$
S=\left\{s\left(a_{j}+r 2^{i}+2^{k}, k\right): 0 \leqq k<j \leqq J, 0 \leqq r<r_{j}\right\}
$$


First it will be seen that for $0 \leqq r<r_{j}$

$$
s\left(a_{j}+(r+1) 2^{i}+2^{k}, k\right) \geqq \frac{1}{2} s\left(a_{j}+r 2^{i}+2^{k}, k\right)
$$

by Lemmas 2 and 4 , and it will be noted that

$$
s\left(a_{j}+\left(r_{j}-1+1\right) 2^{j}+2^{k}, k\right)=s\left(a_{j+1}+02^{j+1}+2^{k}, k\right) .
$$

Second, by Lemma 5 ,

$$
s\left(a_{k+2}+2^{k+1}, k+1\right) \geqq \frac{1}{2} s\left(a_{s}+\left(r_{s}-1\right) 2^{\jmath}+2^{k}, k\right) .
$$

since $n-m>2.2^{J}$ implies $a_{J}+\left(r_{J}-1\right) 2^{j}+2^{k}>m$. Hence the sums in the collection $S$, together with the terms $1, \frac{1}{2}, \frac{1}{4}, \cdots, 1 / 2^{c}$ where $c=\left[\left(\log a_{2}\right)-1\right]$ and the terms $a_{2}^{-1}, a_{3}{ }^{-1}, \cdots, a_{t+1}{ }^{-1},\left(a_{s+1}+1\right)^{-1}, \cdots, n^{-1}$, when ordered as $s_{1} \geqq s_{2} \geqq$ $\cdots \geqq s_{\sigma}$, have the property required by the corollary to Lemma 1 .

Now consider the terms $\frac{1}{3}, \frac{1}{5}, \frac{1}{6}, \cdots 1 /\left(a_{2}-1\right)$ where the missing terms are $1, \frac{1}{2}, \frac{1}{4}, \cdots, 1 / 2^{\mathrm{c}}$. If these are ordered as $t_{1} \geqq t_{2} \geqq \cdots \geqq t_{\rho}$ these have the property required by the corollary to Lemma 1 , and

$$
\sum_{i=1}^{p} t_{j}>\log a_{2}-1-\sum_{j=0}^{c} 1 / 2^{i}>\log a_{2}-3 .
$$

The theorem now follows immediately from the corollary to Lemma 1 and Lemma 6 , for the construction used ensures that the left side of (3) can be written in the form of the left side of (1).

It will be noted that this result is capable of generalization to sums of the iorm $\Sigma \eta_{k} / f(k)$ for functions like $f(n)=n \log n$.

\section{Reference}

R. T. Worley (1976), 'Signed Sums of Reciprocals I', J. Austral. Math. Soc. 21, 410-413.

Jepartment of Mathematics,

Monash University, Jlayton, Victoria 3168,

Australia. 